\title{
The Children's Oncology Group: an opportunity for pediatric radiologists
}

\author{
Ethan A. Smith ${ }^{1}$ \\ Received: 17 July 2019 / Revised: 17 July 2019 / Accepted: 26 July 2019 / Published online: 26 September 2019 \\ (C) Springer-Verlag GmbH Germany, part of Springer Nature 2019
}

The Children's Oncology Group (COG) is a clinical-trialsbased organization focused on childhood and adolescent cancer research. The COG, supported by the National Cancer Institute (NCI), is the largest organization in the world devoted exclusively to pediatric cancer research and has over 200 member institutions, primarily in North America but also in the Netherlands, Switzerland, Australia and New Zealand [1]. At any given time, the COG has multiple active, ongoing clinical trials. In the United States, the extent of the COG's reach is enormous. Each year, greater than $90 \%$ of all newly diagnosed pediatric cancer patients in the United States are cared for at COG member institutions [1].

Fortunately, pediatric cancer is a relatively rare event. With this in mind, researchers quickly recognized that to do effective clinical trials research, they needed a cooperative approach. As early as 1955, there were efforts to pool patient populations and data to more effectively study childhood cancer [2]. Four specific groups emerged by the 1990s, including the Children's Cancer Study Group, Pediatric Oncology Group, National Wilms Tumor Study Group and the Intergroup Rhabdomyosarcoma Study Group [2]. In 2000, these four groups combined to form the Children's Oncology Group $[1,2]$. In its current form, the COG has studies and ongoing clinical trials across the spectrum of pediatric malignancy, from leukemia to central nervous system neoplasms to pediatric solid tumors.

Involvement by radiologists in the COG has been somewhat limited. In the clinical setting, diagnostic imaging is an indispensable part of the care of pediatric cancer patients, both at diagnosis and during treatment as a marker of disease

Ethan A. Smith

Ethan.Smith@cchmc.org

1 Department of Radiology, Cincinnati Children's Hospital Medical Center, University of Cincinnati College of Medicine, 3333 Burnet Ave., MLC 5031, Cincinnati, OH 45229, USA response, stability or progression. Similarly, in clinical trials radiologic studies often serve as the primary biomarker of treatment efficacy, especially in solid tumors. However, despite the importance of imaging in this setting, radiologists themselves often had little or no role in conducting the clinical trials in the past. Most involvement by pediatric radiologists was confined to central imaging review, where a selected radiologist or small group of radiologists would review imaging studies for trial patients and record data, frequently tumor measurements, for a central database. Only rarely were pediatric radiologists involved in the study design, even in areas focused on imaging, where their expertise and input would be valuable.

However, as imaging becomes more complex, with rapidly evolving techniques primarily in MRI and nuclear medicine, the importance of radiologist involvement in COG trials is becoming increasingly clear. The small but consistent Diagnostic Imaging Committee - a recognized COG subcommittee - meets annually at the Children's Oncology Group national meeting. Membership in the Diagnostic Imaging Committee is open to all radiologists with an interest in pediatric cancer imaging. In addition, individual radiologists are now members of multiple tumor-specific subcommittees in COG (for example, the Renal Tumor Committee), usually at the recommendation of the Diagnostic Imaging Committee leadership. Finally, leaders from the Diagnostic Imaging Committee have a seat at the table during COG leadership meetings, and the COG is beginning to recognize that the importance of radiologist involvement extends well beyond central imaging reviews. Increasingly, radiologists are being asked to be more actively involved, including providing input into study design and imaging requirements for trials.

There are three primary incentives for radiologists to be involved in the Children's Oncology Group. First and foremost, involvement affords pediatric radiologists the opportunity to be at the forefront of the team taking care of children and adolescents with cancer, which is in and of itself a noble endeavor. The second major incentive is the potential for 
academic output. The COG is at its core a research group; performing clinical trials and disseminating the work through manuscripts is the purpose of the organization. Academic opportunities for radiologists include not only involvement in manuscripts related to the primary clinical trial goals, but also the ability to perform more limited retrospective spin-off studies through central imaging review. For example, radiologists on the AREN03B2 trial (a renal tumor biology study) have produced several manuscripts over the last few years focused on more limited clinical questions, such as imaging findings of tumor rupture. Finally, involvement in the COG can have more intangible benefits for pediatric radiologists at their home institutions. Relationships with clinical colleagues, mainly pediatric oncologists and surgeons, can be enhanced by recognition that as a radiologist you are engaged and interested in pediatric cancer care enough to be involved in the COG. Face-to-face interactions at the COG meeting, collaborating on conference calls, and participating in study design all serve to enhance the idea that radiologists are clinical and research colleagues, not just an anonymous radiology report.

Beyond ongoing clinical trials involvement, other opportunities are available. One opportunity that is particularly attractive to radiologists is access to the world's largest repository of pediatric cancer imaging studies through the Imaging and Radiation Oncology Core Group (IROC) and Quality Assurance Review Center (QARC). IROC/QARC stores imaging and other data for NCI-sponsored trials, including those done through COG. With emerging capabilities in machine learning and artificial intelligence, the access to such a large dataset of images, large portions of which are already annotated, has the potential to advance pediatric cancer imaging in new and exciting directions.

In summary, the Children's Oncology Group has been a model of success for collaborative research and clinical trials. The work of the COG has advanced pediatric cancer care more rapidly and effectively than would have been possible through the work of individual institutions in isolation. Radiologists are playing an increasingly important role in these clinical trials, both in study design and in central imaging review. Additional academic opportunities exist, and membership and involvement in the COG are keys to accessing the imaging data available. Increasing involvement by pediatric radiologists enhances the clinical and academic goals of the COG and thus will improve the care of pediatric cancer patients throughout the world.

\section{Compliance with ethical standards}

Conflicts of interest None

\section{References}

1. O'Leary M, Krailo M, Anderson JR, Reaman GH (2008) Progress in childhood cancer: 50 years of research collaboration, a report from the Children's Oncology Group. Semin Oncol 35:484-493

2. Children's Oncology Group (2019) "Our History.” Children's Oncology Group website. https://www.childrensoncologygroup. org/index.php/history. Accessed 17 July 2019

Publisher's note Springer Nature remains neutral with regard to jurisdictional claims in published maps and institutional affiliations. 